\title{
A new setup for neutron inelastic cross section measurements
}

\author{
A. Negret ${ }^{1, a, b}$, C. Borcea ${ }^{1,2}$, J.C. Drohé ${ }^{1}$, L.C. Mihailescu ${ }^{1, b}$, A.J.M. Plompen ${ }^{1}$, and R. Wynants ${ }^{1}$ \\ ${ }^{1}$ European Commission, Joint Research Centre, Institute for Reference Materials and Measurements, 2440 Geel, Belgium \\ 2 National Institute for Physics and Nuclear Engineering "Horia Hulubei”, P.O. Box MG-6, Bucharest, Romania
}

\begin{abstract}
An upgraded detection setup for neutron scattering measurements was recently completed at the GELINA neutron source of IRMM. A $200 \mathrm{~m}$ flight path is used. Eight HPGe detectors are placed at $110^{\circ}$ and $150^{\circ}$ with respect to the neutron beam and are read-out by fast digitizers. The neutron flux is monitored by a ${ }^{235} \mathrm{U}$ fission chamber. The first inelastic scattering measurement with the new setup was performed for ${ }^{\text {nat }} \mathrm{Fe}$.
\end{abstract}

\section{Introduction}

In recent years we established that high resolution and high precision gamma-production cross sections can be determined for inelastic scattering and $(\mathrm{n}, 2 \mathrm{n})$ reactions using High Purity Germanium (HPGe) detectors at the $200 \mathrm{~m}$ station at GELINA (Geel Linear Accelerator), the pulsed white neutron source for neutron time-of-flight measurements of JRC-IRMM [1,2]. The study of structural materials needed for applications represents the main interest. Results were obtained for ${ }^{52} \mathrm{Cr},{ }^{58} \mathrm{Ni},{ }^{209} \mathrm{Bi}$ and ${ }^{206,207,208} \mathrm{~Pb}$. They are of importance in the development of accelerator driven systems and the Generation-IV fast reactors. Good accuracy results $(\approx 5 \%)$ were obtained and resonance structure was observed in several cases for the first $\mathrm{MeV}$ above threshold [3].

The measuring time for each of these experiments was typically of the order of two months. An upgrade of the detection setup was carried out in order to decrease this period by

- increasing the number of detectors and,

- increasing the detection efficiency.

\section{Experimental method}

The measurements take place at GELINA [4]. A high intensity pulsed electron beam $(800 \mathrm{~Hz})$ is accelerated to $150 \mathrm{MeV}$ against a thick rotary ${ }^{238} \mathrm{U}$ target. The neutrons are produced via ${ }^{238} \mathrm{U}(\gamma, \mathrm{xn})$ and ${ }^{238} \mathrm{U}(\gamma, \mathrm{f})$ reactions as a result of bremsstrahlung [5]. Although, the target is strongly self-shielding an intense prompt gamma flash is emitted along with the neutrons. The direct neutron flux has a mixed evaporation and fission neutron spectrum that extends from $0.1 \mathrm{MeV}$ to about $20 \mathrm{MeV}$. Outside of this range, the intensity is very low.

A $200 \mathrm{~m}$ flight path is used for measurements of (n,xn $\gamma)$ cross sections. HPGe detectors measure the differential cross sections at $110^{\circ}$ and $150^{\circ}$, which are the nodes of the fourth degree Legendre polynomial. Angle integration is

\footnotetext{
${ }^{a}$ Presenting author,

e-mail: alexandru-liviu.negret@ec.europa.eu

$b$ On leave of absence from National Institute for Physics and Nuclear Engineering "Horia Hulubei”, Bucharest, Romania.
}

performed through a weighted combination of the two angles in a manner that is exact for polynomials of degree six and less. Therefore, accurate angle integrated cross sections are obtained for transitions with multipolarities up to three (E1-3, M1-3). The gamma production cross section is obtained for all visible transitions and for all incident neutron energies. For inelastic scattering typically one gamma-ray is observed for each excited level below 3-4 MeV excitation energy. From the gamma-production cross sections, the total inelastic cross section and the level cross sections are deduced employing an evaluated level scheme for the nucleus of interest. Quasicontinuous excitation curves are obtained with an energy resolution that is in the best cases determined by the $8 \mathrm{~ns}$ time-of-flight resolution of the experiment. This resolution corresponds to the minimal binning of the data required to obtain adequate statistics, but is only marginally worse than the typical time resolution of a HPGe detector.

To obtain the gamma-production cross sections, the detection efficiency, neutron multiple scattering and absorption and flux normalisation have to be determined. The detection efficiency is first measured with a point source. A MCNP simulation is tuned to reproduce the measured efficiency and then a second simulation determines the efficiency corresponding to the extended dimensions of the sample. Corrections for multiple scattering and absorption are made via MCNP simulations. For absolute normalisation of the results, the neutron flux is monitored at the $200 \mathrm{~m}$ cabin by a ${ }^{235} \mathrm{U}$ fission chamber. All measurements are therefore relative to the fission cross section of ${ }^{235} \mathrm{U}$, which is very well known [6].

The main experimental difficulties derive from the low detection efficiency that results in long measuring campaigns. This low detection efficiency is partly due to the use of conventional electronics for signal processing and data-acquisition. Two effects are important. The first is the dead time that results from the very intense gamma flash that precedes every neutron burst. The second is the use of the slow rise time rejection scheme, which is necessary if good time response is to be obtained with large volume HPGe detectors. Finally, of course, the number and size of the detectors is important. One may argue that larger and faster detectors for gamma-rays are available. However, none of these have the 
excellent gamma-ray energy selectivity, which we believe is essential for obtaining accurate $(\mathrm{n}, \mathrm{xn} \gamma)$ cross sections.

\section{Upgrade of the detection system}

Compared to earlier reports, the detection efficiency was improved by increasing the number of detectors and by improving the dead time and time response for each detector by using fast wave form digitizers.

\subsection{Upgrade of the number of detectors}

All measurements mentioned in the introduction were performed with 2-4 HPGe detectors, placed in a horizontal plane around the sample. In order to further increase the number of detectors a support had to be created taking account of the preferred angles (nodes of the fourth Legendre polynomial) and the fact that the forward hemisphere is less favourable due to the enhanced impact of the gamma-flash. In the present experimental arrangement the only cause for observation of the gamma flash and the subsequent long dead time with conventional pulse processing, is the fact that such gammas are scattered by the sample under study. Forward scattering is considerably stronger than backward scattering. As mentioned in the previous section, the HPGe detectors have to be placed at $110^{\circ}$ and $150^{\circ}$ with respect to the incoming beam in order to allow a precise angle integration of the cross section. Therefore, although the angular distribution is symmetric with respect to $90^{\circ}$, the two corresponding forward angles $\left(70^{\circ}\right.$ and $30^{\circ}$ ) were avoided.

An aluminum frame was completed and installed which provides support for twelve HPGe detectors. Four detectors may be supported at each of the angles of $110^{\circ}, 125^{\circ}$ and $150^{\circ}$. The first eight HPGe detectors are currently placed at $110^{\circ}$ and $150^{\circ}$, four in the horizontal and four in the vertical plane. Seven detectors have a relative efficiency of $100 \%$ and one has $70 \%$. The angle of $125^{\circ}$ is of interest only if a better knowledge of the angular distribution of gamma rays is required. A manifold with eight outlets facilitates manual filling of the HPGe detectors with liquid nitrogen.

\subsection{A digitizer-based acquisition system}

The effort for the migration towards a digitized acquisition system started three years ago [7]. The digitized acquisition brings a much simplified electronic scheme together with an improvement of the efficiency due mainly to a better handling of the gamma flash. In addition, for gamma-rays of energy less than $500 \mathrm{keV}$ the time response is significantly improved compared to the conventional slow rise time rejection scheme, resulting in a much improved detection efficiency.

The DC440 digitizers from Acqiris are used. They have 12 bits amplitude resolution, i.e., 4096 channels full range, and a sampling frequency of $420 \mathrm{Msamples} / \mathrm{s}$, i.e., $2.38 \mathrm{~ns} / \mathrm{sample}$. This is sufficient for the energy and time resolution that the HPGe detectors can achieve. The DC440 card has no onboard processing capabilities. To limit data transfer time, two

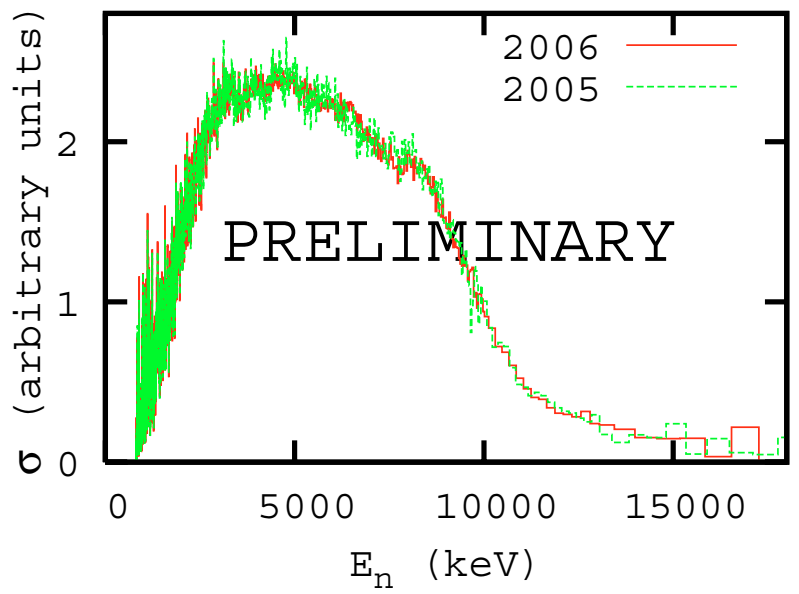

Fig. 1. Total inelastic cross section of ${ }^{206} \mathrm{~Pb}$. Comparison of two independent measurements performed at IRMM in 2005 and 2006 respectively. During the 2006 measurement campaign the new digitized acquisition system was used.

Aquiris compact PCI crates are used. Each has two DC440 cards with two independent channels so that one card serves two detectors. Each crate is connected to a dedicated PC. The acquisition software acquires the traces from the digitizers, performs the online data processing and stores the time and the energy for each event in listmode files.

Each card has an external trigger common to the two detectors that are connected. The trigger is based on a coincidence condition between the $T_{0}$ signal that is synchronous with the electron pulse shortly before it strikes the neutron producing target and a signal coming from any of the two detectors. Detector signals result in a trigger only when they occur at a time corresponding to the neutron energy range of interest. The card is therefore read-out only when a neutron-induced event took place. In that case both channels are read and the two traces $\left(10^{4}\right.$ samples of $\left.2.38 \mathrm{~ns}\right)$ are transferred to the PC. This corresponds to a rate of about 6 events/second for each detector and yields a negligible dead time for the acquisition.

The digitized system was extensively tested before being adopted as a main acquisition system for the upgraded setup [7]. A further check was provided recently with a predecessor of the setup described here. In order to improve the knowledge of the ${ }^{206} \mathrm{~Pb}\left(\mathrm{n}, \mathrm{n}^{\prime}\right)$ and ${ }^{206} \mathrm{~Pb}(\mathrm{n}, 2 \mathrm{n})$ cross sections but also to test the digitizer-based acquisition, an earlier experiment was reiterated using a different (larger) enriched sample and other detector efficiencies. The new measurement benefited from better statistics and the gamma production cross sections for a few extra transitions could be obtained. Figure 1 compares the total inelastic cross section of ${ }^{206} \mathrm{~Pb}$ from the two measurements. Indeed, the results overlap better than the considered uncertainties of 5\%. This comparison brought the final validation of the digitized acquisition system.

Generally, the performances of the digitized acquisition in terms of time and energy resolution are similar to those of the conventional acquisition based on CFDs and ADCs. However, we should note that, in order to meet this goal, a very clean initial signal is required. Special efforts were made in order to avoid any source of electronic noise. Figure 2 displays a region 


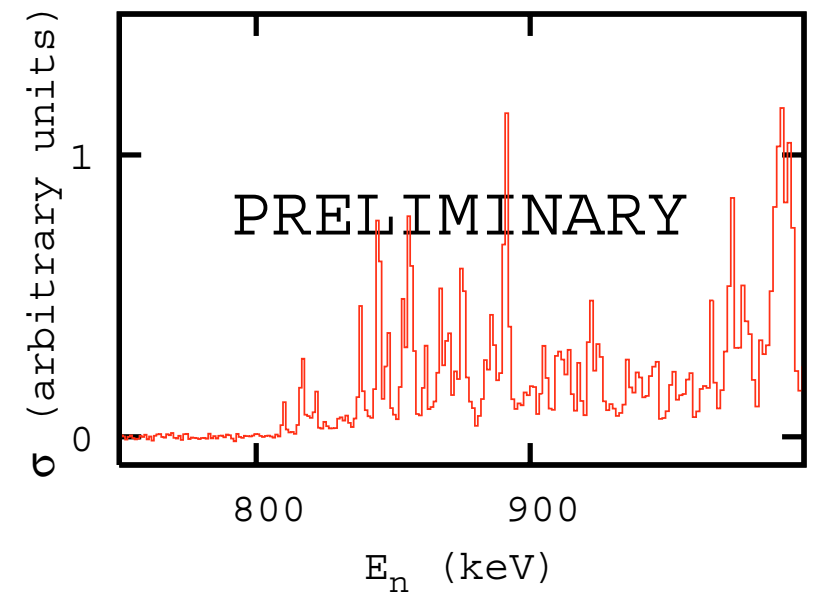

Fig. 2. Zoom of the gamma production cross section for the $803 \mathrm{keV}$ transition in ${ }^{206} \mathrm{~Pb}$. The very good time (i.e., neutron energy) resolution allows us to probe the structure of the compound nucleus.

of the gamma production cross section for the first gamma transition in ${ }^{206} \mathrm{~Pb}$ obtained with the digitizers. The very good time (i.e., neutron energy) resolution allows us to probe the resonant structures.

The two main advantages of the digitized acquisition are the increased detection efficiency and a significant simplification of the required electronic scheme associated with the triggering.

The increase of efficiency comes primarly from a better handling of the gamma flash. With the conventional system, each time when a gamma flash hits a detector and the signal was passed to an ADC, the dead time was of the order of $10 \mu \mathrm{s}$ and blocked the system for the entire neutron burst. This was the case for about $15 \%$ of all bursts. The digitized system avoids this dead time as it is able to disentangle pulses separated by more than $1.5 \mu \mathrm{s}$. Therefore, pulse pile-up of a neutron-induced signal with a gamma-flash induced signal is no longer a problem. It may be noted that the data transfer of 20000 samples $(40 \mathrm{kB})$ per event takes a considerable fraction of the time between two neutron bursts $(1.25 \mathrm{~ms}$ at $800 \mathrm{~Hz}$ burst rate). However, using a trigger that checks if a neutroninduced event occured results in an effective time between two events of about $100 \mathrm{~ms}$. Thus, system dead time is negligible. We use the capability of recording the signal before the trigger. The procedure to improve the time response for gamma-rays with energies less than $500 \mathrm{keV}$ is explained in ref. [7].

The use of digitizers also reduces the additional electronic modules needed. They are limited to the simple trigger scheme mentioned above, while the detector's outputs are provided directly to the digitizers. This becomes important in view of the increased number of detectors used at present, not only in terms of simplification but also for decreasing the chance of malfunction of one of these modules.

\section{Preliminary results}

The first measurements with the new setup were performed on an iron sample. Iron is essential as structural material;

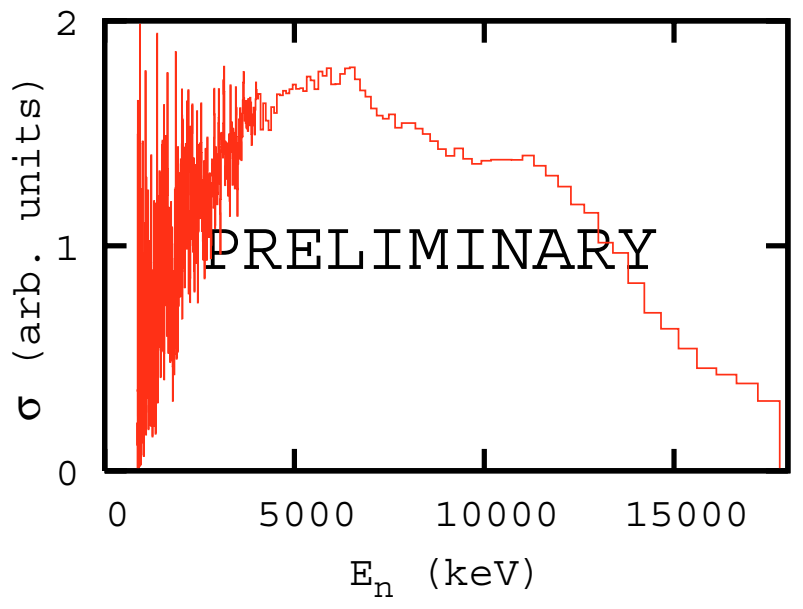

Fig. 3. Total inelastic cross section of ${ }^{56} \mathrm{Fe}$. Preliminary results.

it was emphasized [8] that more precise inelastic and $(n, 2 n)$ cross sections are required in the design of Accelerator-Driven Assemblies.

We used a ${ }^{\text {nat }} \mathrm{Fe}$ sample. The data was accumulated for about six weeks. In order to estimate the background conditions short runs were made with ${ }^{\text {nat }} \mathrm{Ni},{ }^{27} \mathrm{Al}$ and with an empty frame. The analysis of these data is under way. It seems that the new geometry of detection we use, with the frame in place, guarantees rather clean conditions from the point of view of the scattered neutrons or gammas. A first estimate of the shape of the total inelastic cross section of ${ }^{56} \mathrm{Fe}$ is shown in figure 3 .

\section{Summary and outlook}

The setup running at GELINA for $(\mathrm{n}, \mathrm{xn} \gamma)$ measurements underwent recently a major upgrade. The number of HPGe detectors was increased from four to eight and a furter extension to twelve is foreseen in the near future. The acquisition for the HPGe detectors migrated towards one based on digitizers, providing higher efficiency and stability. The digitizer-based acquisition was extensively tested and validated by carefull comparison with the conventional system.

A dedicated software was developed for the new acquisition. Although it is running properly, further software upgrades are required. A more user-friendly interface and better visualisation capabilities have to be implemented. Further, a wider software, dedicated to more than one type of measurement will be developed and adopted.

In conclusion, the new setup existing at the $200 \mathrm{~m}$ cabin of GELINA represents primarily a spectrometer for the study of $(\mathrm{n}, \mathrm{xn} \gamma)$ reactions on structural materials, inert materials and fission products with significantly improved measurement time (about three-five weeks versus the initial ten-twelve weeks). It will represent a "data factory" for inelastic neutron scattering data and will open various possibilities for other applications. The setup may be operated as a user facility for external parties. Data analysis for measurements on natural iron performed with this setup is underway and a measurement campaign was started for natural silicon. 
The authors acknowledge financial support from the integrated project EUROTRANS.

\section{References}

1. L.C. Mihailescu et al., Nucl. Instrum. Meth. A 531, 375 (2004).

2. L.C. Mihailescu et al., Nucl. Phys. A, available online at www.sciencedirect.com (in press).
3. L.C. Mihailescu et al. (these proceedings).

4. D. Tronc et al., Nucl. Instrum. Meth. A 228, 217 (1985).

5. M. Flaska et al., Nucl. Instrum. Meth. A 531, 394 (2004).

6. P. Obložinský, M. Herman, Nucl. Data Sheets 107, 2931 (2006).

7. L.C. Mihailescu et al., Nucl. Instrum. Meth. Phys. Res. A (for publication).

8. G. Aliberti, G. Palmiotti, M. Salvatores, C.G. Stenberg, Nucl. Sci. Eng. 146, 13 (2004). 